\title{
Stability, magnetic order, and electronic properties of ultrathin $\mathrm{Fe}_{3} \mathrm{O}_{4}$ nanosheets
}

\author{
Pär A. T. Olsson $\odot,{ }^{1,2,{ }^{*}}$ Lindsay R. Merte $\odot,{ }^{1}$ and Henrik Grönbeck ${ }^{3}$ \\ ${ }^{1}$ Materials Science and Applied Mathematics, Malmö University, SE-205 06 Malmö, Sweden \\ ${ }^{2}$ Division of Mechanics, Lund University, SE-221 00 Lund, Sweden \\ ${ }^{3}$ Department of Physics and Competence Centre for Catalysis, Chalmers University of Technology, SE-412 96 Gothenburg, Sweden
}

(Received 9 January 2020; revised manuscript received 29 March 2020; accepted 31 March 2020; published 23 April 2020)

\begin{abstract}
We study the stability, magnetic order, charge segregation, and electronic properties of a novel three-layered $\mathrm{Fe}_{3} \mathrm{O}_{4}$ film by means of Hubbard-corrected density functional theory calculations. The stable film is predicted to consist of close-packed iron and oxygen layers, comprising a center layer with octahedrally coordinated $\mathrm{Fe}$ sandwiched between two layers with tetrahedrally coordinated Fe. The film exhibits an antiferromagnetic type I spin order. A charge analysis confirms that the stable structure has distinct charge segregation, with $\mathrm{Fe}^{2+}$ ions in the center layer and $\mathrm{Fe}^{3+}$ in the tetrahedral surface layers. Examination of the electronic band structures and densities of states shows that the bandgap is substantially reduced, from $2.4 \mathrm{eV}$ for the bulk rocksalt to $0.3 \mathrm{eV}$ for the film. The reduction in the bandgap is a consequence of the $2+$ to $3+$ change in oxidation state of Fe in the surface layers.
\end{abstract}

DOI: 10.1103/PhysRevB.101.155426

\section{INTRODUCTION}

Ultrathin transition metal oxide films are of significant interest for a wide range of intriguing applications in different disciplines, ranging from heterogeneous catalysis [1-3] to spintronics [4] and advanced electronics [5-7]. Many of the attractive physical and chemical characteristics of thin metal oxide films can be attributed to the reduced coordination at the surfaces, which, thanks to the high surface-to-volume ratio, dominate the systems and give rise to properties that deviate substantially from those of the bulk. The properties of the films can be further tailored by the interaction with metal substrates, which alter the coordination and bonding and thereby affect the overall physical properties [7-10]. This would in principle provide a means for designing, or customizing, composite systems comprising a transition metal oxide film and an appropriate metal support to meet the desired properties for specific applications. Successful examples along this line of research include the combination of an $\mathrm{FeO}(111)$ monolayer on a $\mathrm{Pt}(111)$ substrate, for which it has been found that the low-temperature catalytic activity for $\mathrm{CO}$ was improved compared to that of pure $\mathrm{Pt}(111)$ [9-13].

Because the adhesive bonding between the $\operatorname{Pt}(111)$ substrate and the $\mathrm{Fe}^{2+}$ cations is relatively strong, other more weakly interacting substrates have been used to investigate the impact of the adhesion on the catalytic performance. Suitable candidates for this purpose are silver substrates, which are

*par.olsson@mau.se

Published by the American Physical Society under the terms of the Creative Commons Attribution 4.0 International license. Further distribution of this work must maintain attribution to the author(s) and the published article's title, journal citation, and DOI. Funded by Bibsam. relevant mainly for two reasons: (i) they interact weakly with both $\mathrm{Fe}$ and $\mathrm{O}$ and (ii) exhibit a low work function and electronegativity, which reduces the charge transfer between the film and the substrate. Through a series of recent experiments, the existence of a novel three-layered $\mathrm{Fe}_{3} \mathrm{O}_{4}$ phase grown on a $\operatorname{Ag}(100)$ substrate has been confirmed [14,15]. This appears to be a distinct meta-stable form of $\mathrm{Fe}_{3} \mathrm{O}_{4}$ with a thickness of about $1 \mathrm{~nm}$. The structure consists of three close-packed $\mathrm{FeO}(111)$ layers that are arranged such that the top and bottom layers are terminated by close-packed $\mathrm{O}$ layers. Similarly to previously characterized thin $\mathrm{FeO}$ films [16], the presence of both $\mathrm{Fe}^{2+}$ and $\mathrm{Fe}^{3+}$ ions has been observed [14]. Such mixed valence is in contrast to bulk $\mathrm{FeO}$, which only contains $\mathrm{Fe}^{2+}$ [17], but necessary to ensure the charge neutrality of $\mathrm{Fe}_{3} \mathrm{O}_{4}$. Moreover, the new structure shows mixed coordination: it comprises one close-packed Fe layer with octahedral coordination at the center of the film and two close-packed Fe layers with $\mathrm{Fe}$ tetrahedrally coordinated next at the film/substrate and film/gas-phase interfaces. Such surface layer arrangements are of the same tetrahedral/wurtzite type as previously reported for rocksalt $\mathrm{FeO}$ on $\mathrm{Ru}(0001)$ [18,19] and $\mathrm{CoO}$ on $\operatorname{Ir}(100)$ [20-22]. However, the layer stacking sequence is different from that in previous reports [18-22], as the stacking faults effectively cancel when the structure is only three layers thick. Thus, the new phase has a tetrahedral-octahedral-tetrahedral (TOT) layer combination with the close-packed Fe layers ordered in an $A B C$ stacking sequence.

Thanks to the reduced dimension, the overall physical properties are likely strongly affected by the local coordination of the surface layers. Hence, it is possible that the heterogeneous character of the film, in combination with the high surface-to-bulk ratio, gives rise to electronic bandgaps and magnetic orderings that differ from those of the most common bulk phases. Moreover, in light of the limited 
thickness, it is reasonable to assume that the phase is at least partially stabilized by surface effects such as surface energy and stress. To quantify these surface effects we resort to density functional theory (DFT) modeling.

The purpose of the present study is threefold: (i) to characterize the stability of the novel three-layered $\mathrm{Fe}_{3} \mathrm{O}_{4}$ phase, (ii) to investigate the ground-state magnetic structure and charge segregation in the low-temperature limit, and (iii) to characterize the associated electronic properties.

To gain insight into (i), we calculate how the total energy varies with the lattice parameter for different surface termination types. For (ii) we investigate the impact of the magnetic orderings on the total energy. We find that the TOT structure with Fe tetrahedrally coordinated at the surfaces and octahedrally coordinated in the center of the film is energetically preferred. This film has an antiferromagnetic (AFM) type I spin ordering. Through a Bader charge analysis [23] we find that the $\mathrm{Fe}$ ions in the center layer correspond to $\mathrm{Fe}^{2+}$, while the ions at the surfaces are of the $\mathrm{Fe}^{3+}$ type. For (iii) we investigate the electronic band structure and density of states (DOS) for different types of surface terminations and make comparisons with the rocksalt bulk phase. It is found that the surface terminations with tetrahedrally coordinated $\mathrm{Fe}$ give rise to substantial reductions in the bandgap compared with films with octahedral rocksalt (111) terminations and bulk rocksalt.

\section{METHODS}

\section{A. Density functional theory calculations}

To investigate the stability, magnetic order, and electronic properties of the iron oxide films by means of Hubbardcorrected spin-polarized DFT $+U$ modeling, we use the Vienna $a b$ initio simulation package (VASP) [24-27]. The interaction between the valence electrons and the core is described by the projector augmented wave (PAW) method [28,29]. The electronic valence configurations are given by $2 s^{2} 2 p^{4}$ $(\mathrm{O}), 3 p^{6} 3 d^{7} 4 s^{1}(\mathrm{Fe})$, and $4 p^{6} 4 d^{10} 5 s^{1}(\mathrm{Ag})$. The exchangecorrelation functional is described by the generalized gradient approximation using the Perdew, Burke, and Ernzerhof (PBE) formula [30,31]. Because noncollinear components have been found to be small for $\mathrm{FeO}$ [19], we model the spins collinearly. The electronic wave functions are expanded in plane waves and the reciprocal space is discretized by means of a $\Gamma$ centered $k$-point grid generated using the Monkhorst-Pack scheme [32]. To ensure well-converged results, we studied how the total energy of the stoichiometric bulk rocksalt $(\mathrm{FeO}$, wüstite, or B1) phase changes with the kinetic energy cutoff and $k$-point grid size. For this purpose, $\mathrm{FeO}$ is represented by a hexagonal supercell as in Refs. [33,34] with an AFM type II spin variation along the [111] direction. It was found that using a plane-wave cutoff of $650 \mathrm{eV}$ and $k$-point grid of $12 \times 12 \times 3$ yields well-converged results with a total energy accuracy within $5 \mathrm{meV} / \mathrm{FeO}$, which is of sufficient accuracy for the present investigation.

To account for the strong electronic correlation of the $\mathrm{Fe} 3 d$ states, we utilize the rotationally invariant Hubbard correction approach of Dudarev et al. [35]. In this approach, the effective Hubbard parameter $U_{\text {eff }}$ is specified, which is the difference between the on-site Coulombic $(U)$ and the effective on-site exchange parameter $(J)$. In the literature, numerous different $U_{\text {eff }}$ values have been employed for modeling iron oxides, typically ranging from 3.6 to above $5.0 \mathrm{eV}$ [8,33,34,36-41]. We evaluated the bandgap of bulk $\mathrm{FeO}$ for values of $U_{\text {eff }}$ in the range $3.6-4.5 \mathrm{eV}$ and found that this property increases monotonically from 1.9 to $2.6 \mathrm{eV}$, with an approximate agreement with the experimental value of $\sim 2.4 \mathrm{eV}$ for $U_{\text {eff }}=4.2 \mathrm{eV}$ [42]. This $U_{\text {eff }}$ value was adopted for the rest of the calculations. The corresponding lattice parameter for $\mathrm{FeO}$ was found to vary only slightly: from 4.40 to $4.43 \AA$ for the considered range of $U_{\text {eff }}$ values. The lattice parameter is calculated to be $4.42 \AA$ for $U_{\text {eff }}=4.2 \mathrm{eV}$, which is an overestimation of the experimental value of $4.33 \AA[43,44]$ by about $2 \%$. The overestimation is in line with general observations for the PBE functional [45]. The choice of the effective Hubbard parameter was further validated by comparing the computed bandgap for the $\mathrm{Fe}_{2} \mathrm{O}_{3}$ hematite phase with experimental data. The calculated value of $1.8 \mathrm{eV}$ is in good agreement with the experimental range 1.9-2.2 eV [46].

To gain insight into the charge distribution in the considered surfaces, we perform a Bader charge analysis [23] using the open-source software BADER [47]. As references, we compare the results with stoichiometric rocksalt, hematite, and $\mathrm{Fe}_{3} \mathrm{O}_{4}$ magnetite bulk phases, which enables us to relate the ionic types in the films to those in the bulk phases.

\section{B. Model systems}

The aim of this study is to model $\mathrm{Fe}_{3} \mathrm{O}_{4}$ films, which experimentally are grown on $\operatorname{Ag}(100)$ substrates. The film has a small lattice mismatch with respect to $\operatorname{Ag}(100)$, giving rise to an extended moiré-type supercell. However, to reduce the computational cost associated with the DFT $+U$ calculations, we consider the unsupported films. The motivation behind this simplification is the assumption that the interaction between the support and the film is weak. To investigate the interaction, we computed the adhesion energy between different types of $\mathrm{Fe}_{3} \mathrm{O}_{4}$ films and $\mathrm{Ag}(100)$ and $\operatorname{Ag}(111)$ supports using DFT $+U$ in conjunction with the semiempirical Grimme-D2 van der Waals correction [48] to account for dispersion interactions. The results revealed that the adhesion energies typically lie in the range 0.2-0.4 eV/ $/ \mathrm{Fe}_{3} \mathrm{O}_{4}$. Moreover, a Bader charge analysis showed only a small charge transfer of 0.1 electron/ $/ \mathrm{Fe}_{3} \mathrm{O}_{4}$ from the support to the films. The low adhesive energy and modest charge transfer are in accordance with previous DFT $+U$ results for $\mathrm{FeO}$ monolayers on $\mathrm{Ag}(100)$ supports and validates the use of freestanding films [8].

The considered film structures are constructed with alternating $\mathrm{Fe}$ and $\mathrm{O}$ layers. Each layer consists of two $\mathrm{Fe}$ or $\mathrm{O}$ atoms, yielding a total of six $\mathrm{Fe}$ atoms and eight $\mathrm{O}$ atoms. To preserve the symmetry of the close-packed layers and coherency across the periodic boundaries, the slab is represented by a rectangular supercell with the ratio of the inplane lattice parameters fixed, such that $a_{0}=b_{0} / \sqrt{3}$. A planar $k$-point mesh corresponding to $10 \times 6 \times 1$, is used together with dipole correction to remove dipole interaction across the vacuum [49]. To ensure that the films are not affected by the periodicity, the total energy is converged with respect 
(a)
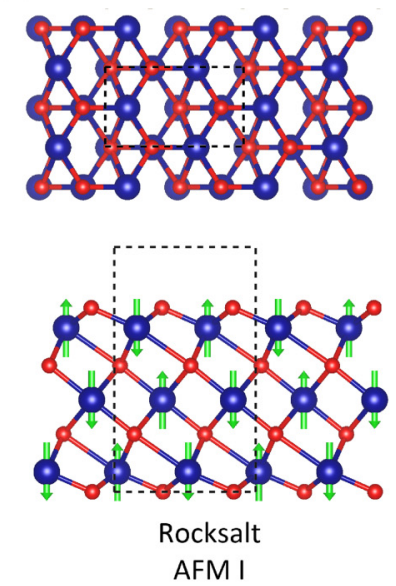

(d)
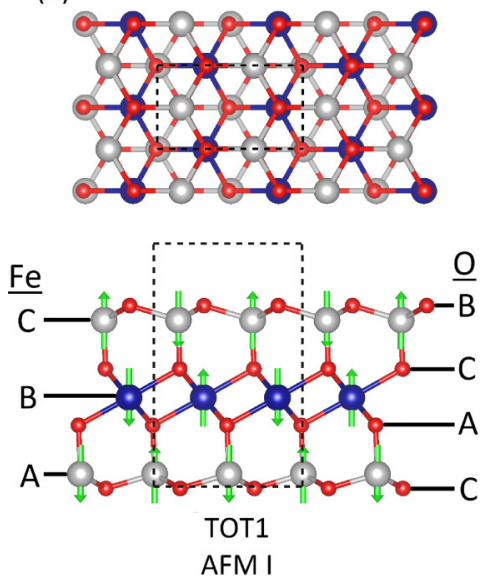

(g)
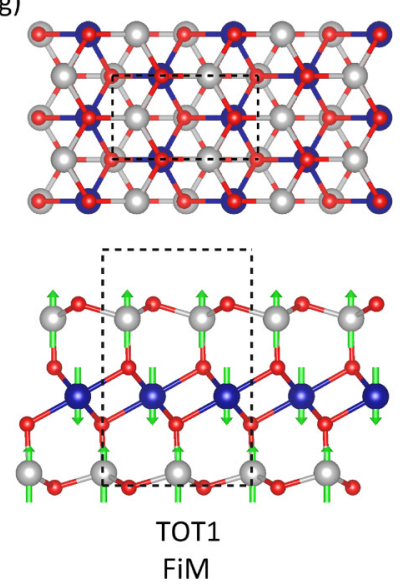

(b)
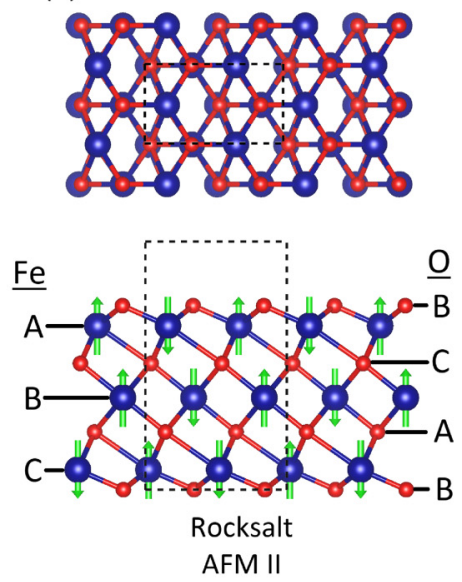

(e)

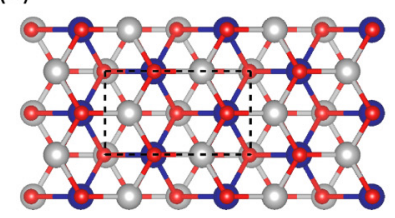

(c)
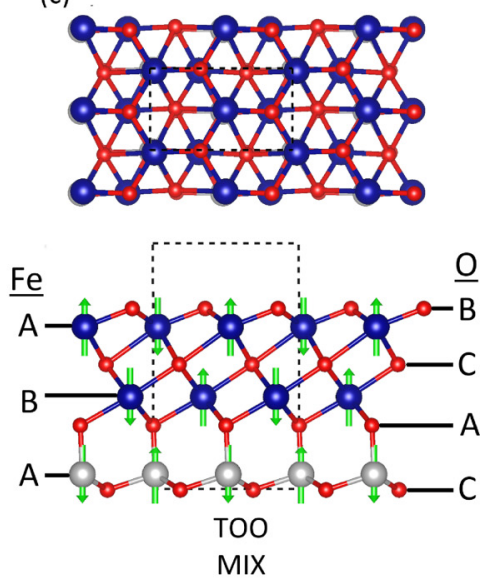

(f)

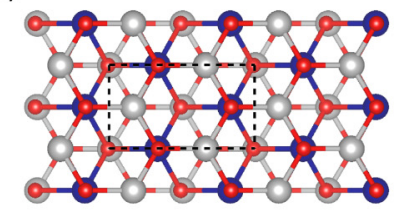

(h)
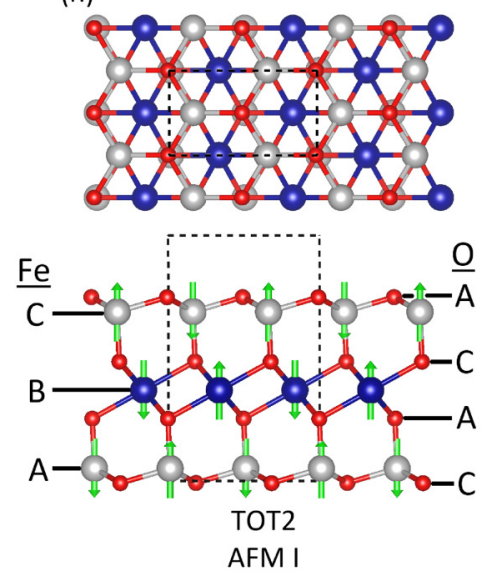

(i)
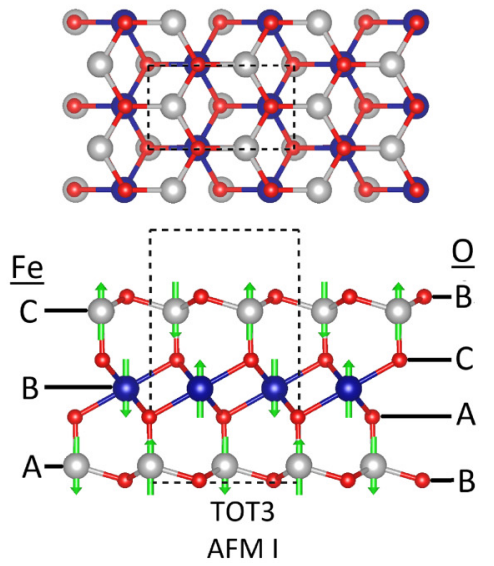

FIG. 1. Structures and magnetic orderings for (a) rocksalt AFM I, (b) rocksalt AFM II, (c) TOO mix, (d) TOT1 AFM I, (e) TOT1 AFM II, (f) TOT1 mix, (g) TOT1 FiM, (h) TOT2 AFM I, and (i) TOT3 AFM I. Upper and lower panels in each case show the top and side view, respectively. Arrows represent the up and down spin associated with the Fe ions and dashed rectangles indicate unit cells. Atomic color code: $\mathrm{Fe}$ in octahedral position (blue), Fe in tetrahedral position (silver), and oxygen (red). Stacking sequences are indicated in (b)-(d), (h), and (i).

to the out-of-plane vacuum. The results show that a vacuum thickness of $6 \AA$ or larger yields a convergence accuracy of $1 \mathrm{meV} / \mathrm{Fe}_{3} \mathrm{O}_{4}$. Hence, we make sure that a vacuum larger than $8 \AA$ is used in the calculations.

We study three types of three-layered films: (i) rocksalt with surface $\mathrm{Fe}$ atoms on octahedral coordination [see
Figs. 1(a) and 1(b)]; (ii) rocksalt with one surface having $\mathrm{Fe}$ ions in tetrahedral coordination, i.e., tetrahedral-octahedraloctahedral (TOO) stacking [see Fig. 1(c)]; and (iii) the TOT structure [see Figs. 1(d)-1(i)]. It should be noted that we also tried to study a three-layered film with a tetrahedraltetrahedral-octahedral (TTO) stacking order. However, this 
structure turned out to be unstable and relaxed without barrier to the TOO structure. Because the $O$ layers of the TOT structure can have different stacking orders, we identify three types: TOT1 [see Figs. 1(e)-1(g)], TOT2 [see Fig. 1(h)], and TOT3 [see Fig. 1(i)]. The respective stacking sequences of the O layers for the different types correspond to $C A C B, C A C A$, and $B A C B$.

\section{MAGNETIC ORDER}

The magnetic structure of rocksalt $\mathrm{FeO}$ has an AFM II order below the Néel temperature $(\sim 200 \mathrm{~K}$ for $P=0)$. However, as discussed in Refs. [18,19], the local spin structure may differ in the proximity of surfaces with Fe ions in tetrahedral positions. Thus, to identify the lowest-energy configuration, we investigate different spin orderings.

For the rocksalt structure, we consider the AFM I and AFM II types [see Figs. 1(a) and 1(b), respectively]. Because the stacking symmetry is broken in the TOO structure, the lowest-energy spin ordering is of mixed type as depicted in Fig. 1(c). For the TOT1 phase, we examine two AFM spin combinations: AFM I and AFM II [see Figs. 1(d) and 1(e), respectively]. Moreover, a mixed configuration, which can be regarded as AFM I with the spins of $\mathrm{Fe}$ atoms in the center layer having the same spin orientation [see Fig. 1(f)]. This type of ordering is analogous to that found for the $\mathrm{FeO}$ and CoO wurtzite terminations previously modeled with DFT + $U[18,19,21]$. The two final configurations for TOT1 are a ferrimagnetic (FiM) structure [see Fig. 1(g)] and a ferromagnetic (FM) configuration where all the magnetic moments associated with the $\mathrm{Fe}$ atoms have the same direction (not shown in Fig. 1). For the TOT2 and TOT3 phases, we only examine the AFM I type [see Figs. 1(h) and 1(i), respectively].

\section{RESULTS AND DISCUSSION}

\section{A. Stability and magnetic order}

To study the structure and magnetic order that minimize the total energy for the TOT1 structure, we computed the energy for the five aforementioned spin configurations as a function of the in-plane lattice parameters. The spin configuration is found to have a small influence on the equilibrium lattice parameter, which is calculated to be $a_{0}=3.21 \AA$ for all cases. This value is $2.2 \%$ larger than the experimental value of $3.14 \AA[15]$ and consistent with the overestimation of the bulk lattice constant.

The stable magnetic order for TOT1 is AFM I. The computed total energies relative to the AFM I configuration for the remaining magnetic configurations are $+0.10,+0.05$, +0.15 , and $+0.40 \mathrm{eV} / \mathrm{Fe}_{3} \mathrm{O}_{4}$ for AFM II, mixed, FiM, and FM, respectively. Thus, the preferential magnetic ordering deviates from that found for bulk rocksalt. The in-plane lattice parameter is larger than the lattice constant computed for bulk $\mathrm{FeO}$, which is $3.12 \AA$. This is an indication that the phase undergoes an expanding in-plane relaxation when the free surfaces are introduced. Because surface elastic components of a close-packed (111) surface are isotropic [50], the observed positive in-plane relaxation strain shows that the surface stress components are tensile in character [51,52]. The optimized interplanar distance between the neighboring Fe layers of the

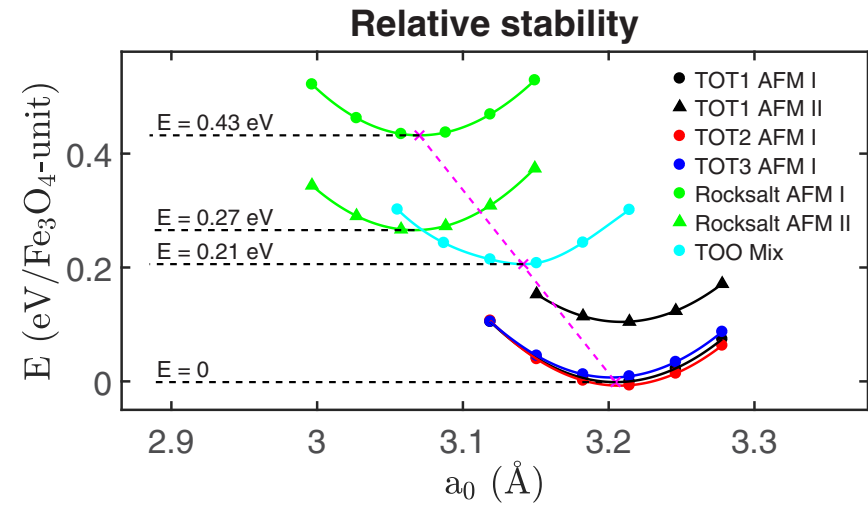

FIG. 2. Relative stability of three-layered slabs with different types of surface terminations. The TOT1 AFM I phase is chosen as reference. The corresponding lattice parameter for bulk rocksalt is $3.12 \AA$. The dashed curve is a guide for the eye that connects the minima for the rocksalt, TOO, and TOT1 AFM I films.

AFM I configuration is $2.91 \AA$, which is in good agreement with the experimental value of about $2.9 \AA$ obtained from surface $x$-ray diffraction measurements [15].

In Fig. 2 we show the total energy as a function of the in-plane lattice parameter for TOT1 AFM I and AFM II together with the remaining morphologies and magnetic orders. Among the considered morphologies, the TOT AFM I is found to be the stable configuration. The energy difference with respect to the TOO and rocksalt films is calculated to be +0.21 and $+0.27 \mathrm{eV} / \mathrm{Fe}_{3} \mathrm{O}_{4}$, respectively. The energy curves for the TOT1-TOT3 phases differ only marginally. Hence, the impact of the stacking sequence of the $\mathrm{O}$ layers on the total energy is small. Previous experimental results suggest that the X-ray rod profiles for the TOT1 phase are in very good agreement with the DFT $+U$ data [15], whereas those of the TOT2 and TOT3 deviate somewhat. However, because of the similar energies of the TOT2 and TOT3 structures, we cannot exclude them based only on energetic arguments. Therefore there is a possibility that separate islands could assume different $\mathrm{O}$ stacking sequences.

Unlike the TOT phases, the rocksalt film has an AFM II magnetic order, which is the same type as observed for bulk rocksalt. The AFM I configuration is about $0.16 \mathrm{eV} / \mathrm{Fe}_{3} \mathrm{O}_{4}$ higher in energy. The likely cause behind the change in preferential magnetic order is a superexchange mechanism, where the AFM coupling is dictated by the Fe-O-Fe connectivity [53]. This is altered when transforming the rocksalt to TOT films, such that the angles of the Fe-O-Fe bridges change from $90^{\circ}$ and $180^{\circ}$ to about $120^{\circ}$. As the strength of the superexchange mechanism depends greatly on the $\mathrm{Fe}-\mathrm{O}-\mathrm{Fe}$ angles, typically being strongest at $180^{\circ}$ and weakest at $90^{\circ}$, the AFM I preference is most likely a consequence of Fe ions preferably assuming tetrahedral positions in the surface.

The magnetic ordering does not have any impact on the ground-state in-plane lattice parameter of rocksalt films, which is found to be $3.07 \AA$. This is a decrease compared to the corresponding value for bulk rocksalt $\mathrm{FeO}$, which suggests that the surfaces with $\mathrm{Fe}$ ions in octahedral positions induce a negative biaxial relaxation strain on the phase. Thus, 
TABLE I. Magnetic moments and Bader charges associated with Fe sites in the bulk and ultrathin film. The Bader charge is computed relative to the neutral $\mathrm{Fe}$ atom and given as the elementary charge. Magnetic moments are given in units of Bohr magnetons, $\mu_{B}$.

\begin{tabular}{|c|c|c|c|c|c|c|}
\hline & \multicolumn{2}{|c|}{ Bulk tetrahedral site } & \multicolumn{2}{|c|}{ Bulk octahedral site } & & \\
\hline & $\begin{array}{l}\text { Magnetic } \\
\text { moment }\end{array}$ & $\begin{array}{l}\text { Bader } \\
\text { charge }\end{array}$ & $\begin{array}{l}\text { Magnetic } \\
\text { moment }\end{array}$ & $\begin{array}{l}\text { Bader } \\
\text { charge }\end{array}$ & & \\
\hline Rocksalt & - & - & $\pm 3.64-3.69$ & +1.35 & & \\
\hline Hematite & - & - & \pm 4.22 & +1.82 & & \\
\hline \multirow[t]{2}{*}{ Magnetite } & +4.07 & +1.68 & $-3.66 /-4.17$ & $+1.40 /+1.78$ & & \\
\hline & $\begin{array}{l}\text { Magnetic } \\
\text { moment }\end{array}$ & $\begin{array}{l}\text { Bader } \\
\text { charge }\end{array}$ & $\begin{array}{l}\text { Magnetic } \\
\text { moment }\end{array}$ & $\begin{array}{l}\text { Bader } \\
\text { charge }\end{array}$ & $\begin{array}{l}\text { Magnetic } \\
\text { moment }\end{array}$ & $\begin{array}{l}\text { Bader } \\
\text { charge }\end{array}$ \\
\hline Rocksalt & - & - & \pm 4.16 & +1.76 & \pm 3.72 & +1.36 \\
\hline TOO & \pm 4.04 & +1.66 & \pm 4.15 & +1.76 & \pm 3.66 & +1.41 \\
\hline
\end{tabular}

unlike the surfaces with Fe in tetrahedral positions, the surface stresses are compressive in character.

The difference in the lattice parameter for films with $\mathrm{Fe}$ ions in tetrahedral and octahedral positions correlate with the charge state of the Fe ions in the surface layers. The Bader charge (see Table I) of the Fe ions located in octrahedral postions is calculated to be $+1.76 e$ with respect to the neutral atom. This is larger than the corresponding value for Fe ions in tetrahedal positions, which is calculated to be $+1.66 e$. Thus, the Fe ions in octrahedral positions are smaller than those in tetrahedal position.

The TOO phase can be viewed as a hypothetical intermediate phase between the rocksalt and the TOT structures, where one of the surfaces of the rocksalt morphology has been transformed from having the Fe ions in octahedral coordination to tetrahedral. This causes a disruption in the stacking sequence, which leads to a mixed type of magnetic order that is an intermediate configuration of the AFM I and AFM II types. The minima in the potential energy curves for the AFM I-ordered rocksalt, TOO, and TOT films lie on a straight line (dashed line in Fig. 2) such that the TOO ground-state energy and in-plane lattice parameter $(3.14 \AA)$ are the averages of those of the TOT and rocksalt. This behavior suggests that the ground-state energy can be described in terms of surface contributions augmented with the bulk contribution when the spin order is the same.

Based on the calculations, it can be concluded that the TOT AFM I morphology is preferred among the considered configurations. It should be noted that also the total energies of the FiM, AFM II, and mixed spin configurations are lower than the energies of the rocksalt and TOO films. These findings confirm that the atomic geometry associated with tetrahedral surface terminations is preferential, which is in agreement with recent experimental observations $[14,15]$. Thus, it is concluded that the phase stability of the TOT phase can be attributed to two mechanisms: (i) the reduced surface energy associated with the geometrical organization of the atoms associated with tetrahedrally coordinated surface layers and (ii) the reduction in surface energy owing to the transforma- tion in magnetic ordering from AFM II to AFM I, where the former represents the largest contribution to the stability.

\section{B. Charge segregation and magnetic moments}

To characterize the charge segregation and magnetic moments of the films we performed a Bader charge analysis and present the charges together with the magnetic moments of the ground-state structures in Table I. For comparison, we also present results for the bulk phases.

The computed magnetic moments for bulk rocksalt, 3.6$3.7 \mu_{B}$, are found to agree well with available experimental results, being in the range $\pm 3.3-4.2 \mu_{B}[44,54,55]$. The Bader charge analysis reveals that the Fe ions in octahedral positions are $\mathrm{Fe}^{1.4+}$ ions, with respect to the neutral atom. This corresponds to a formal charge of $\mathrm{Fe}^{2+}$. The magnetic order of the hematite structure corresponds to an AFM order along the [0001] direction with alternating magnetic moments of $4.2 \mu_{B}$ (see Table I). This result slightly underestimates the experimentally observed magnetic moments, which are in the range 4.6-4.9 $\mu_{B}[56,57]$. Based on the Bader charge analysis, we find that the charge associated with the Fe sites in hematite is +1.8 , which is associated with a formal charge of $\mathrm{Fe}^{3+}$.

The magnetite phase is a spinel structure where two-thirds of the Fe ions are octahedrally coordinated and one-third are tetrahedrally coordinated [17]. Below the Verwey temperature $(\sim 120 \mathrm{~K})$, the tetrahedrally coordinated $\mathrm{Fe}$ atoms are of the $\mathrm{Fe}^{3+}$ type, whereas half of the octahedral sites are $\mathrm{Fe}^{3+}$ and half are $\mathrm{Fe}^{2+}$. The $\mathrm{Fe}$ ions in tetrahedral positions exhibit magnetic moments of $4.1 \mu_{B}$, and the charge is calculated to be $\mathrm{Fe}^{1.7+}$, which should be associated with the formal $\mathrm{Fe}^{3+}$ ions. The $\mathrm{Fe}$ ions in octahedral positions have magnetic moments of $-3.7 \mu_{B}$ and $-4.1 \mu_{B}$ and the respective Bader charges are $+1.4 e$ and $+1.8 e$, which correspond to formal states of $\mathrm{Fe}^{2+}$ and $\mathrm{Fe}^{3+}$, respectively. These results compare well with previous DFT $+U$ reports [58].

As shown in the lower portion of Table I, the magnetic moments $\left(\sim 3.7 \mu_{B}\right)$ and Bader charges $(+1.4 e)$ associated with the octahedrally coordinated $\mathrm{Fe}$ in the center of the film 

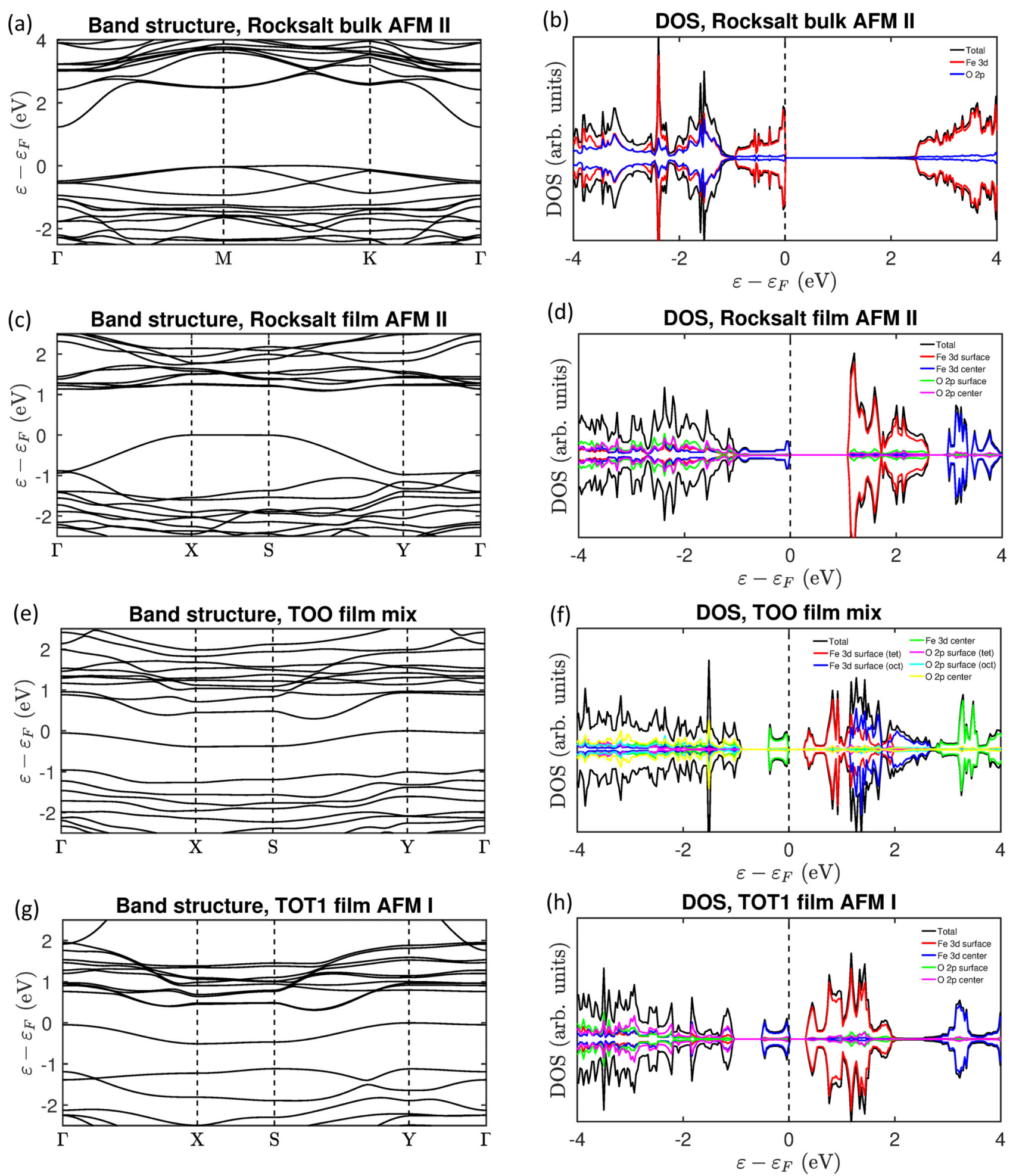

FIG. 3. Band structure, projected DOS, and total DOS for (a), (b) bulk rocksalt and slab geometries (c), (d) rocksalt AFM II, (e), (f) TOO mix, and (g), (h) TOT1 AFM I. The reference energy is the Fermi energy for all systems and the planar $k$-point paths in the left panel correspond to those of hexagonal and orthorhombic unit cells as proposed in Ref. [59].

concur with the data for bulk octahedral sites in rocksalt and the $\mathrm{Fe}^{2+}$ sites in magnetite. This suggests that the center $\mathrm{Fe}$ ions are of the $\mathrm{Fe}^{2+}$ type. The octahedrally coordinated $\mathrm{Fe}$ ions in the surfaces of rocksalt and TOO have Bader charges and magnetic moments that match those of octahedral $\mathrm{Fe}^{3+}$ sites in magnetite and hematite. The magnetic moments and charges associated with the tetrahedral surface Fe ions are in line with the results for the tetrahedral sites in magnetite and in good agreement with those found for hematite, which suggests that these sites should be associated with $\mathrm{Fe}^{3+}$. Thus, the ion type associated with $\mathrm{Fe}$ atoms at the surfaces is $\mathrm{Fe}^{3+}$, regardless of the type of surface termination, whereas $\mathrm{Fe}$ 
atoms in the center layer correspond to $\mathrm{Fe}^{2+}$. These findings are in line with the experimental observations of mixed charge segregation found for the TOT phase in previous $\mathrm{x}$-ray photoelectron spectroscopy measurements [14].

\section{Electronic properties}

To investigate the electronic properties of the rocksalt, TOT1, and TOO films, we compute the electronic band structures together with the total and projected DOS. The results are compared to $\mathrm{FeO}$ bulk in the rocksalt structure (see Fig. 3). The computed DOS for bulk $\mathrm{FeO}$ resembles those previously reported using the DFT $+U$ approach $[33,34,60]$. Inspection of the band structure for bulk rocksalt shows that there is a parabolic band with a minimum at the $\Gamma$ point, which reduces the bandgap to less than $2.4 \mathrm{eV}$ [see Fig. 3(a)]. This is mainly owing to Fe $4 s$ states emerging between the Fe $d$-valence and conduction bands [61,62]. The contribution of the $4 s$ states to the total electronic DOS is minor [see Fig. 3(b)].

There is a reduction in the bandgap to about $1.1 \mathrm{eV}$ for the rocksalt film [see Figs. 3(c) and 3(d)]. The main reason for the reduction is the change in valence of the $\mathrm{Fe}$ ions at the surface layers, which shifts the unoccupied states to lower energies. There is also a positive energy shift of $\mathrm{Fe} 3 d$ states that are initially below the Fermi level for the bulk phase but are shifted above the Fermi level for the film. These changes in DOS are consistent with the change in oxidation states from $\mathrm{Fe}^{2+}$ to $\mathrm{Fe}^{3+}$.

The size of the bandgap is further reduced by the introduction of tetrahedrally coordination surface layers in the TOO and the TOT films [see Figs. 3(e)-3(h)]. Based on analysis of the DOS, the valence band associated with centered Fe $3 d$ states is narrowed and localized to about $0.5 \mathrm{eV}$ below the Fermi level. The conduction states associated with the surface Fe atoms are shifted to lower energies, which narrows the bandgap to about $0.3 \mathrm{eV}$.

These findings show that the film remains semiconducting, although the bandgap is significantly reduced as a result of the $\mathrm{Fe}$ ions in tetrahedral positions in the surface layers. In comparison, wurtzite-terminated $\mathrm{CoO}$ surfaces, characterized by scanning tunneling microscopy, have been proposed to obtain metallic properties [20-22]. This is believed to occur as a result of polarity compensation, which is manifested as a change in the surface oxidation states [63], similar to that observed in the present case. For the considered films, we observe nonnegligible potential differences between the center layer and the surface layers, suggesting interlayer polarity for which the tetrahedral geometry could serve to compensate.
However, the presence of $\mathrm{Fe}^{3+}$ in the surface layers itself acts to compensate this polarity [63]. It is therefore unclear to what extent polarity affects the coordination geometry.

\section{CONCLUSIONS}

In the present work we have investigated the stability, magnetic order, charge segregation, and electronic properties of three-layered $\mathrm{Fe}_{3} \mathrm{O}_{4}$ films by means of DFT $+U$ calculations. It is found that the TOT structure, with Fe ions in octahedral positions in the center of the film, sandwiched between two layers with $\mathrm{Fe}$ ions in tetrahedral positions, has the lowest energy of all considered morphologies. The stability of the structure is mainly due to the low surface energy associated with having the surface $\mathrm{Fe}$ ions occupying tetrahedral coordinated sites. Moreover, an additional lowering of the total energy is found when the spin organization is of the AFM I type. This is in contrast to bulk rocksalt and rocksalt films, which have an AFM II spin order. Thus, the reduced surface energy, jointly caused by the tetrahedral atomic configuration and its associated magnetic ordering, leads to the stabilization of the three-layered films.

In accordance with the experimental observations, the $\mathrm{Fe}$ ion type found in the three-layered film varies depending on its location in the film. The results of the Bader charge analysis show that the $\mathrm{Fe}$ ions in tetrahedral positions in the surface correspond to $\mathrm{Fe}^{3+}$, which is the same ion type as found in bulk hematite and the tetrahedral sites of magnetite. The ions in the octahedral positions in the center layer are of the $\mathrm{Fe}^{2+}$ type, which concurs with those in bulk rocksalt and half of the octahedral sites in magnetite.

Finally, we study the electronic band structure and DOS for the three-layered structures. It is found that the bandgap is substantially reduced compared to that of the bulk rocksalt, with the largest reduction calculated for the terminations with $\mathrm{Fe}$ ions in tetrahedral positions. This bandgap reduction is in line with the observed change in oxidation states.

\section{ACKNOWLEDGMENTS}

This research was funded by the Swedish Research Council (VR) through Grant Nos. 2016-04162 (P.A.T.O.), 2018-05374 (L.R.M.), and 2016-05234 (H.G.). The DFT $+U$ calculations were performed using computational resources provided by the Swedish National Infrastructure for Computing (SNIC) at the National Supercomputer Centre (NSC), Linköping University, and at the High Performance Computing Center North (HPC2N), Umeå University.
[1] J. Schoiswohl, M. Sock, Q. Chen, G. Thornton, G. Kresse, M. G. Ramsey, S. Surnev, and F. P. Netzer, Top. Catal. 46, 137 (2007).

[2] H.-J. Freund and G. Pacchioni, Chem. Soc. Rev. 37, 2224 (2008).

[3] N. Nilius, Surface Sci. Rep. 64, 595 (2009).

[4] E. Kan, M. Li, S. Hu, C. Xiao, H. Xiang, and K. Deng, J. Phys. Chem. Lett. 4, 1120 (2013).
[5] N. Setter, D. Damjanovic, L. Eng, G. Fox, S. Gevorgian, S. Hong, A. Kingon, H. Kohlstedt, N. Y. Park, G. B. Stephenson et al., J. Appl. Phys. 100, 051606 (2006).

[6] S. D. Ha and S. Ramanathan, J. Appl. Phys. 110, 071101 (2011).

[7] T. Yang, T. T. Song, M. Callsen, J. Zhou, J. W. Chai, Y. P. Feng, S. J. Wang, and M. Yang, Adv. Mater. Interfaces 6, 1801160 (2019). 
[8] L. R. Merte, C. J. Heard, F. Zhang, J. Choi, M. Shipilin, J. Gustafson, J. F. Weaver, H. Grönbeck, and E. Lundgren, Angew. Chem. Int. Ed. 55, 9267 (2016).

[9] Y.-N. Sun, Z.-H. Qin, M. Lewandowski, E. Carrasco, M. Sterrer, S. Shaikhutdinov, and H.-J. Freund, J. Catal. 266, 359 (2009).

[10] Y.-N. Sun, L. Giordano, J. Goniakowski, M. Lewandowski, Z.H. Qin, C. Noguera, S. Shaikhutdinov, G. Pacchioni, and H.-J. Freund, Angew. Chem. Int. Ed. 49, 4418 (2010).

[11] J. Goniakowski and C. Noguera, Phys. Rev. B 79, 155433 (2009).

[12] L. Giordano, M. Lewandowski, I. M. N. Groot, Y.-N. Sun, J. Goniakowski, C. Noguera, S. Shaikhutdinov, G. Pacchioni, and H.-J. Freund, J. Phys. Chem. C 114, 21504 (2010).

[13] Q. Fu, W.-X. Li, Y. Yao, H. Liu, H.-Y. Su, D. Ma, X.-K. Gu, L. Chen, Z. Wang, H. Zhang et al., Science 328, 1141 (2010).

[14] L. R. Merte, M. Shipilin, S. Ataran, S. Blomberg, C. Zhang, A. Mikkelsen, J. Gustafson, and E. Lundgren, J. Phys. Chem. C 119, 2572 (2015).

[15] L. R. Merte, P. A. T. Olsson, M. Shipilin, J. Gustafson, F. Bertram, C. Zhang, H. Grönbeck, and E. Lundgren, J. Chem. Phys. 152, 114705 (2020).

[16] S. Gota, E. Guiot, M. Henriot, and M. Gautier-Soyer, Phys. Rev. B 60, 14387 (1999).

[17] G. S. Parkinson, Surface Sci. Rep. 71, 272 (2016).

[18] L. Martin-Garcia, I. Bernal-Villamil, M. Oujja, E. Carrasco, R. Gargallo-Caballero, M. Castillejo, J. F. Marco, S. Gallego, and J. de la Figuera, J. Mater. Chem. C 4, 1850 (2016).

[19] I. Bernal-Villamil and S. Gallego, Phys. Rev. B 94, 075431 (2016)

[20] W. Meyer, D. Hock, K. Biedermann, M. Gubo, S. Müller, L. Hammer, and K. Heinz, Phys. Rev. Lett. 101, 016103 (2008).

[21] K. Heinz and L. Hammer, J. Phys. Condens. Matter 25, 173001 (2013).

[22] K. Biedermann, M. Gubo, L. Hammer, and K. Heinz, J. Phys. Condens. Matter 21, 185003 (2009).

[23] R. Bader, Atoms in Molecules: A Quantum Theory (Clarendon Press, Oxford, UK, 1994).

[24] G. Kresse and J. Hafner, Phys. Rev. B 47, 558 (1993).

[25] G. Kresse and J. Hafner, Phys. Rev. B 49, 14251 (1994).

[26] G. Kresse and J. Furthmüller, Phys. Rev. B 54, 11169 (1996).

[27] G. Kresse and J. Furthmüller, Comput. Mater. Sci. 6, 15 (1996).

[28] P. E. Blöchl, Phys. Rev. B 50, 17953 (1994).

[29] G. Kresse and D. Joubert, Phys. Rev. B 59, 1758 (1999).

[30] J. P. Perdew, K. Burke, and M. Ernzerhof, Phys. Rev. Lett. 77, 3865 (1996).

[31] J. P. Perdew, K. Burke, and M. Ernzerhof, Phys. Rev. Lett. 78, 1396 (1997).

[32] H. J. Monkhorst and J. D. Pack, Phys. Rev. B 13, 5188 (1976).

[33] M. Cococcioni and S. de Gironcoli, Phys. Rev. B 71, 035105 (2005)

[34] T. Eom, H.-K. Lim, W. A. Goddard, and H. Kim, J. Phys. Chem. C 119, 556 (2015).
[35] S. L. Dudarev, G. A. Botton, S. Y. Savrasov, C. J. Humphreys, and A. P. Sutton, Phys. Rev. B 57, 1505 (1998).

[36] A. Bengtson, D. Morgan, and U. Becker, Phys. Rev. B 87, 155141 (2013).

[37] J. Noh, O. I. Osman, S. G. Aziz, P. Winget, and J.-L. Bredas, Sci. Technol. Adv. Mater. 15, 044202 (2014).

[38] Y. Meng, X.-W. Liu, C.-F. Huo, W.-P. Guo, D.-B. Cao, Q. Peng, A. Dearden, X. Gonze, Y. Yang, J. Wang et al., J. Chem. Theory Comput. 12, 5132 (2016).

[39] N. Y. Dzade, A. Roldan, and N. H. de Leeuw, Minerals 4, 89 (2014).

[40] M. Busch, V. Mehar, L. R. Merte, M. Shipilin, E. Lundgren, J. F. Weaver, and H. Grönbeck, Chem. Phys. Lett. 693, 84 (2018).

[41] M. Shipilin, E. Lundgren, J. Gustafson, C. Zhang, F. Bertram, C. Nicklin, C. J. Heard, H. Grönbeck, F. Zhang, J. Choi et al., Top. Catal. 60, 492 (2017).

[42] H. Bowen, D. Adler, and B. Auker, J. Solid State Chem. 12, 355 (1975).

[43] E. R. Jette and F. Foote, J. Chem. Phys. 1, 29 (1933).

[44] H. Fjellvåg, F. Grønvold, S. Stølen, and B. Hauback, J. Solid State Chem. 124, 52 (1996).

[45] P. Haas, F. Tran, and P. Blaha, Phys. Rev. B 79, 085104 (2009).

[46] K. Sivula, F. Le-Formal, and M. Grätzel, ChemSusChem 4, 432 (2011).

[47] W. Tang, E. Sanville, and G. Henkelman, J. Phys.: Condens. Matter 21, 084204 (2009).

[48] S. Grimme, J. Comput. Chem. 27, 1787 (2006).

[49] J. Neugebauer and M. Scheffler, Phys. Rev. B 46, 16067 (1992).

[50] V. B. Shenoy, Phys. Rev. B 71, 094104 (2005).

[51] R. Dingreville, J. Qu, and M. Cherkaoui, J. Mech. Phys. Solids 53, 1827 (2005).

[52] P. A. T. Olsson and H. S. Park, J. Mech. Phys. Solids 60, 2064 (2012).

[53] R. Cornell and U. Schwertmann, The Iron Oxides: Structure, Properties, Reactions, Occurrences and Uses (Wiley, New York, 2006).

[54] W. L. Roth, Phys. Rev. 110, 1333 (1958).

[55] P. D. Battle and A. K. Cheetham, J. Phys. C: Solid State Phys. 12, 337 (1979).

[56] J. M. D. Coey and G. A. Sawatzky, J. Phys. C: Solid State Phys. 4, 2386 (1971).

[57] E. Kren, P. Szabo, and G. Konczos, Phys. Lett. 19, 103 (1965).

[58] H. Liu and C. Di Valentin, J. Phys. Chem. C 121, 25736 (2017).

[59] W. Setyawan and S. Curtarolo, Comput. Mater. Sci. 49, 299 (2010).

[60] B. Himmetoglu, A. Floris, S. de Gironcoli, and M. Cococcioni, Int. J. Quantum Chem. 114, 14 (2014).

[61] I. I. Mazin and V. I. Anisimov, Phys. Rev. B 55, 12822 (1997).

[62] G. Trimarchi, Z. Wang, and A. Zunger, Phys. Rev. B 97, 035107 (2018).

[63] J. Goniakowski and C. Noguera, Phys. Rev. B 83, 115413 (2011). 